\title{
الاستفادة من نظام غزل القطن ( الغزل الحلقى ) فى انتاج خيط زخرفى من خلط القطن مع البولى اكريليك
}

Utilization of cotton yarn system (Ring Spinning) in producing Fancy Yarn of blend cotton with poly acrylic

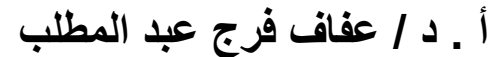
أستاذ بقسم الغزل والنسيج والتريكو - كلية الفنون التطبيقية - جامعة حلو المطان

Prof. Afaf Farag

Spinning, Weaving, and Knitting Dept.- Faculty of Applied Arts - Helwan University afaffarag1999@yahoo.com م. د / ايمن السيد ابراهيم رمضان

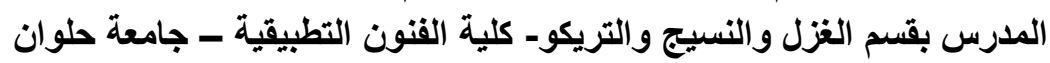

Lect. Ayman Elsayed Ebrahim Ramdan

Lecturer, Department of Spinning, Weaving and Knitting - Faculty of Applied Arts Helwan University aymaneisayd2664@yahoo.com الباحث/ خليل ابراهيم ابراهيم مهندس غزل ونسيج وتريكو بشركة النصئ

Researcher. Khalel Ibrahim

Student at the Department of Spinning, Weaving and Knitting - Faculty of Applied Arts - Helwan University khalelibrahim632@gmail.com

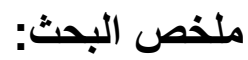
نظر الأن صناعة الغزل والنسيج من الصناعات التنافسية التي تعتمد على الإنتاج بشكل مبتكر، وبأفضل جودة ممكنة، وتقليل التكلفة حتى يتم الاستمر ار فى التنافس وتصبح السلع أكثر مبيعا. لذا كان النظر إلى تقليل التكلفة والاستفادة فى إنى

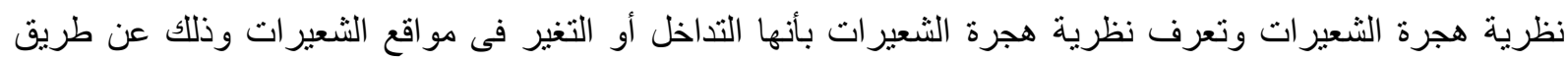
إمكانية إنتاج خيط على ماكينة الغزل الحلقى من مبرومين أكريليك و القطن بنسب مختلفة، ويكون للمنتج النهائى القدرة التنافسية التى تجعله المنتج المفضل لدى المستهلك، ـ ومن الضرورى عند إجراء عمليات الخلط اختيار أفضل الخلطات للحصول على نتائج جيدة على ضوء الخواص المطلوبة في الخيوط المخلوطة. ومن هنا يمكن تلخيص مشكلة البحث في كيفية الحصول علي أفضل مواصفة خيط نتيجة خلط مبرومين من القطن والاكريليك، ـ ولتحقيق هذا الهدف تم إنتاج عدد (9)عينات مخلوطة بين خامتي القطن و الأكريليك بنظام القطن أثناء مرحلة الغزل النهائى، ذلك للحصول علي خيوط ذات خو اص جديدة، وقد تم تنفيذها على ماكينات غزل حلقى (تيودا) مع عمل تعديل فى مسار الخيط، و تعتمد منهجية البحث على المنهج التجريبي و التحليلى والربط بين كل منهما، وقد أسفرت الدر اسة عن بعض النتائج أهمها: 1 ـ كلما زادت نمرة الخيط قلت قراءات قوة الثد و الحمل القاطع، و عند زيادة نسبة الخلط بالقطن زادت قوة الثند والحمل القاطع . 2 - كلما زادت النمرة ( ترقيم انجليزي ) كلما قلت النسبة المئوية للاستطالة، وعند زيادة نسبة القطن في تكوين الخيط 


$$
\begin{aligned}
& 3 \text { - كلما كانت نمرة الخيط رفيعة كلما زادت عدم الانتظامية و عدد العقد و التشعير . } \\
& 4 \text { - كلما زادت نسبة خلط القطن في الخيط الناتج زادت الانتظامية وقلت عدد العقد و التشعير . } \\
& \text { الكلمات المفتاحية : } \\
& \text { ( خلط الخيوط - خيط زخرفى- هجرة الثعبرات ). }
\end{aligned}
$$

\section{Abstract:}

Since the textile industry is one of the competitive industries that depend on innovative production, at the best possible quality, and reducing costs until competition continues and goods are sold more. So the consideration was to reduce cost and benefit in the theory of capillary migration and the difference in the theory of capillary migration as interference or change in the location of capillaries through the possibility of producing a thread on the spinning machine of acrylic and cotton twins in different proportions, and the final product has the competitive ability that makes it the preferred product of the consumer ,. It is necessary when performing mixing operations to choose the best mixtures to obtain good results in light of the required properties in the blended strands. From here, we can summarize the research problem in how to obtain the best thread specification as a result of mixing brominated cotton and acrylic. To achieve this goal, a number (9) samples were mixed between the cotton and acrylic materials in the cotton system during the final spinning stage, in order to obtain threads with new properties. It was implemented on machines for spinning (tioda) with an amendment in the thread path, and a methodology is adopted Research on the experimental and analytical approach and the link between both of them, the study has yielded some results, the most important of which are:-

1. The higher the thread count, the lower the tensile strength and the cutter load. When the cotton mixing ratio increases, the tensile strength and the cutter load increase.

2. The higher the tigress (English numbering), the lower the percentage of elongation, and when the percentage of cotton in the thread formation increases, the elongation ratio decreases.

3. The higher the thread, the more the irregular the number of nodes and the notification .

4. The higher the proportion of cotton mixing in the resulting thread, the greater the uniformity and the fewer the number of nodes and the hair.

\section{Keywords :}

(Blend yarns - Fancy yarn - Migration of the fibers).

تنتأثر خواص الأقمشة المخلوطة بدرجة كبيرة بنوعية الثعبرات المستخدمة والنسبة المئوية لكل منهما فى الخلطة حيث توجد مشكلات عديدة فى إنتاج خلطة ذات خواص وظيفية أو جمالية نتناسب مع طبيعة استخدام هذه الأقشة.

و المشكلة الأساسية فى الخلط هو نوزيع الثعيرات المخلوطة فى الخيط، ومدى علاقة نوزيع الثعيرات فى الخيط

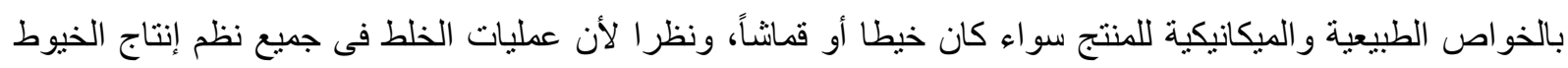
تتم فى مرحلة السحب حيث يتم خلط الخامات فى صورة أثرطة، .لهذا كان اختيار البحث ينحصر فى نظام الخلط حيث يتم الخلط على ماكينة غزل الألياف القطنية، حيث إن ألياف البولى اكريليك يتم غزلها على أطو الها ( 10سم ) وخلطها مع لته القطن (35 مم )، وبذلك ممكن دراسة ظاهرة هجرة الثعبرات ونظام ترتيب وتوزيع الشعير ات بالخيوط المنتجة بنظام 
1 - الاستفادة من خلط القطن مع الألياف البولى اكريليك على ماكينات غزل القطن لار اسة سلوك الشعير ات وتوزيعها فى

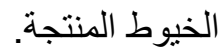

2 - إستخدام نسب خلط مختلفة من القطن مع ثبات نسبة خلط الألياف البولى اكريليك للحصول على خيوط زخرفية . 3 - در اسة ظاهرة هجرة الثعبيرات لمعرفة مدى تأثثير ها على الخو اص الطبيعية والميكانيكية للخيوط المنتجة.

1 - استخدام الخيوط المنتجة فى إنتاج أقشة ملابس شتوية تتاسب جميع الأعمار من الجنسين.

2 - الحصول على أقششة ذات جودة عالية ترضى أذواق المستهلكين.

3 - إثراء المكتبة العربية بمر اجع عن ظاهرة هجرة و إنتاج الخيوط الزخرفية بخامات و ألوان ونسب خلط مختلفة مع تغيير نظم الخلط.

1 - يفترض البحث أنه يمكن الاستفادة من نظرية هجرة الثعيرات فى خلط ألياف القطن مع الألياف البولى اكريليك في إنتاج خيوط زخرفية باستخدام نظام القطن. 2 - الخلط بين مبرومين ( خامات طبيعية ، خامات صناعية ) على ماكينة الغزل الحلقى بنظام القطن بؤثر على خواص

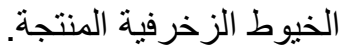
3 - خلط خامات مختلفة ( طبيعية + صناعية ) ذات ألوان مختلفة على ماكينة الغزل الحلقى بنظام القطن يؤدى الى إنتاج

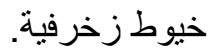
منهج البحث:• يستخدم فى البحث المنهج التجريبى و المنهج التحليلى

الخيط المخلوط الزخرفى هو الخيط الناتج عن تغذية مبرمين أو أكثر مجتمعين معا بواسطة مجموعة من البرمات فى

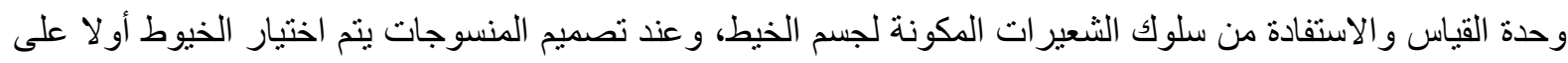
أساس مواصفاتها الميكانيكية مثل القوة، المرونة ...الخ ، ويمكن أيضا الاختيار على أساس ما يسمى بالمواصفات الوظيفية مثل نفاذية الماء و الهواء، ـوتتحدد المواصفات الميكانيكية والوظيفية أساسا حسب نوع الخيط، نمرة الخيط، برم الخيطو نظام الغزل المستخدم في الإنتاج ،ولكن قد يتم اختيار الخيوط بسبب مظهرها، بحيث بمكن عمل أنواع خاصة من لن الخيوط سو اء المفردة أو المزوية لإعطاء تأثير ات زخرفية الإناج كنية معينة (9).

: 1 تعرف عملية الخلط فى صناعة الغزل والنسيج بأنها تجميع ألياف ذات خواص مختلفة معًا في عمل خيوط لإنتاج أقمشة مخلوطة خلطًا جيدًا ومتجانسًا من ألياف مختلفة. كما تعرف الخيوط المخلوطة بأنها الخيوط التى تصنع من نوعين أو أكثر من الثعيرات أو الألياف النسيجية المختلفة

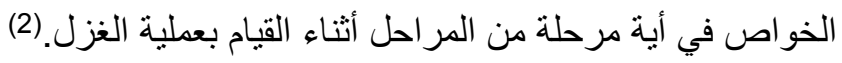


تنركز الأهداف الرئيسية لعملية الخلط في تقليل تكلفة الخيوط المنتجة والحصول على خواص جيدة ومرغوبة لمواجهة

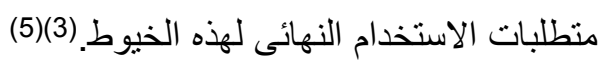

$$
\text { : } 1
$$

يطلق مصطلح الخيوط الزخرفية على الخيوط التى تحمل تأثثرات خاصة وقد يكون هذا الخيط مفرداً أو مزوياً ويمكن إضافة هذه التأثيرات أثناء عملية الغزل أو عملية الزوى أو بعد إنتاج الخيط أثناء عملية الصباغة أو الطباعة (12).

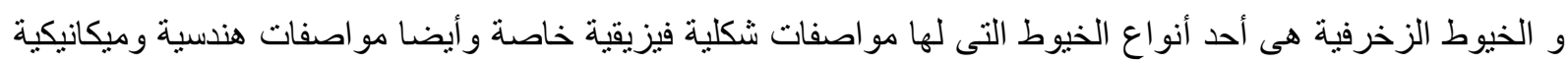

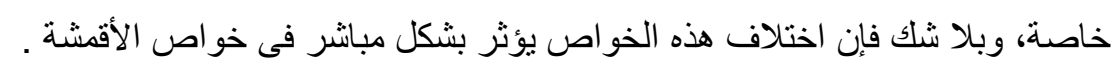

: 1 The Core or Base Yarn 1 - خيط الأساس ( الأرضي) - 1 التركي)

2 - خيط الزخرفة The Effect or Fancy Yarn

3 - خيط الربط

: 1

1. خيوط ذات مناطق سميكة 1 Slub Yarn

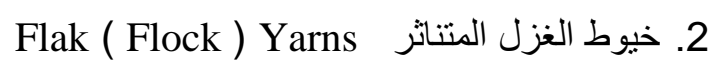

3. الخيوط ذات السحاب Cloud Yarns

4. خيوط النيكروبوكر Knickerbockers Yarns

5lame Yarns الخيوط ذات الشعلة

6.

7. الخيوط ذات العقد مeb Yarns

8. الخيوط المعدنية Mallic yarns

9. الخيوط ذات البذور Splash and Seed Yarns

10. الخيوط ذات العراوي Loop (Boucle) Yarns

11. الخيوط ذات العقدات Ratine Yarns

12. الخيوط ذات التشابكات Snarl Yarns

13. خيوط الجرانديل 11

14. خيوط الثينيل 15

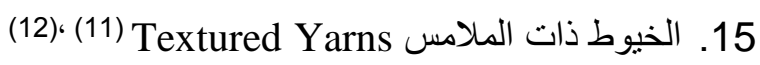

1 - 4 - ظاهرة هجرة الشعيرات:

لقد اتجه العديد من الباحثين إلى دراسة ظهرة الظاهرة حسب كيفية دراسته لها و النظريات التى افترضت واستخدمت وتم تجربتها للوصول إلى دراسة كافية عن تلك الك الظاهرة)(4). و اعتبر "بيرز" Peire (13) إن هجرة الثعيرات هى التداخل أو التغير فى مواقع الثعيرات، وقد أرجع هذه الظاهرة إلى التشابك العشو ائى للشعيرات بالخيط. 
بينما اعتبر "هامليتون" Hamilton (16) أن هجرة الشعيرات هى اصطلاح يستخدم للالالة على حركة الشعيرات فى الخيط، وذلك بالقياس لمحور الخيط (1)، كذلك وجد أن خلط الثعيرات غير المتشابهة فى الخيوط المغزولة من المحتمل أن

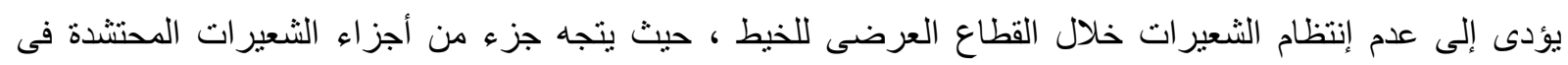

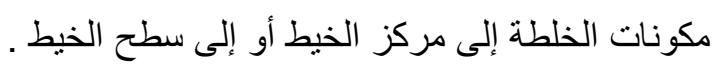

وتوضح النتائج التى تم الحصول عليها من بحوث ( تويند Townend) إلى أن الهجرة ترجع إلى أكبر مساحة تلامس للسطح (13)،(7) ، ولذا فإنه فى الخلطات المكونة من شعيرات طويلة وقصيرة أو شعيرات رفيعة وسميكة خلال عملية

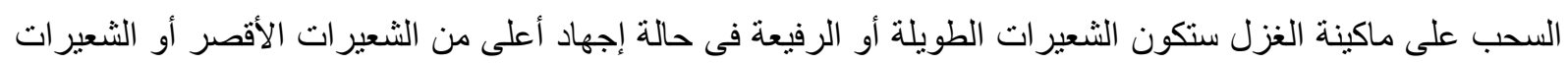

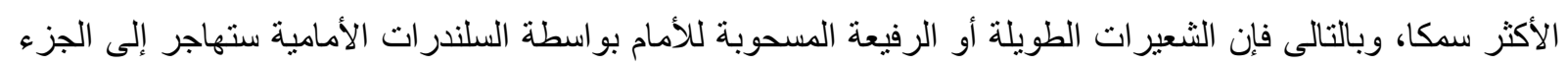

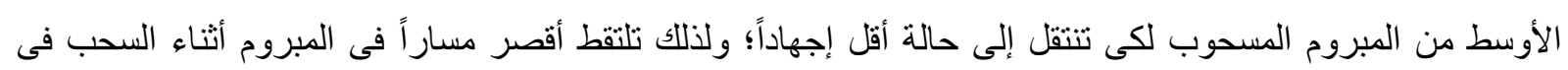
الغزل النهائى (15) ، وقد استخدم " ميرشنت وآخرون Merchant el al (17) اصطلاح التكوم Drif دلالة على لهئ الهجرة.

و اعتبر سكاردينو" Scardino "ليونز" Lyons إنه للحصول على نوزيع متجانس للشعيرات شكل (1)،أو من أجل توزيع عشوائى لعناصر الخلطة فإن منوسط النسبة للعنصر فى أية منطقة يجب أن تكون نفس النسبة للعنصر فى أية منطقة يجب أن تكون نفس النسبة للعنصر فى أنحاء الخيط ككل (14).

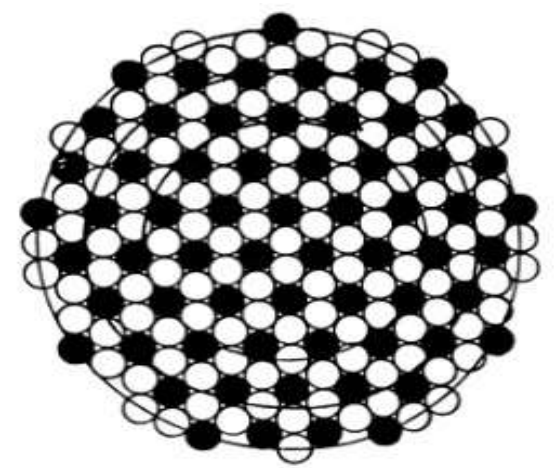

شكل(1) الثكل يوضح القطاع العرضى المثالى للخيط المخلوط حيث الثعيرات تأخذ الثكل الدائرى المنتظم وموزعة خلال مناطق متعادلة فى المساحة

$$
2 \text { - } 2 \text { - الإطار التطبيقي للبحث :- التجارب النسجية :- }
$$

تم إنتاج عدد (9) عينات مختلفة تحت الدراسة فى مصنع الغزل بشركة النصر للغزل و النسيج و التريكو "الثوربجى" بالمو اصفات التالية :1. مبروم من القطن أطوال شعير اته 35 مم مخلوط مع مبروم من ألياف البولى اكريليك بأطوال شعيرات 100 مم على

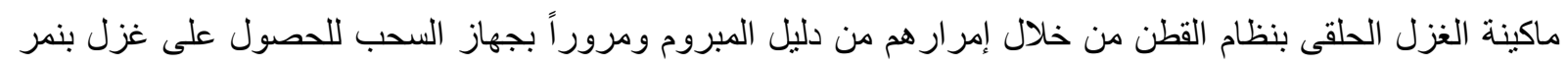
خيط 7 / و آخر 1/10 وثالث 1/14 بترقيم القطن، وذللك بخلط بنسبة ( 1:1 ) بو اقع مبروم واحد قطن : مبروم واحد بولى أكريلك . 2. مبروم من القطن اطو ال شعيرات 35 مم مخلوط مع مبروم من الياف البولى أكريليك بأطو ال شعيرات 100 مم على ملى

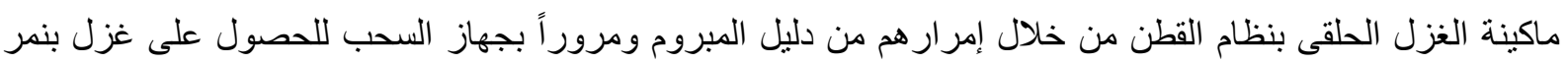


المجلد الخامس - العدد الثاني والعشرون

خيط 7 / وآخر 1/10 وثالث 1/14 بترقيم القطن، وذلك بخلط بنسبة ( 1:2 ) بو اقع مبرومين قطن : مبروم واحد بولى أكريلك.

3. مبروم من القطن اطو ال شعيرات 35 مم مخلوط مع مبروم من ألياف البولى أكريليك بأطو ال شعيرات 100 مم على

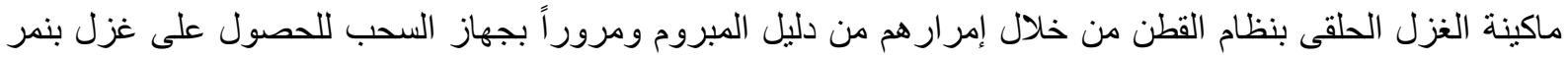
خيط 7 / و آخر 1/10 وثنالث 1/14 بترقيم القطن، وذللك بخلط بنسبة ( 1:3 ) بو اقع عدد 3 مبروم قطن : مبروم واحد بولى أكريليك .

2 - 2 - أولا: مواصفات ألياف القطن جيزة 86 المستخدمة فى التجارب ( 12 ) :

جلول مواصفات ألياف القطن جيزة 86 المستخدمة فى التجارب

\begin{tabular}{|c|c|c|c|c|c|}
\hline القيمة & البيــــان & b & القيمة & البيـــــــــان & م \\
\hline 86.0 & نسبة انتظامية الشعيرات \% & 5 & 33.2 & طول الثُعيرة / مم & 1 \\
\hline 6.4 & نسبة استطالة الشعيرات \% & 6 & 5.5 & معامل اختلاف طول الثعيرة \% & 2 \\
\hline \multirow[t]{2}{*}{7.5} & نسبة الرطوبة \% & 7 & 9.38 & منانة الشعير ات / برسلي & 3 \\
\hline & & & 4.6 & دقة الثعير ات / ميكرونير & 4 \\
\hline
\end{tabular}

2 - 3 - ثانيا: مواصفات ألياف البولى أكريليك المستخدمة فى التجارب ( 13 ) :

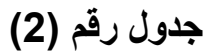
مواصفات ألياف البولى أكريليك المستخدمة فى التجارب

\begin{tabular}{|c|c|c|}
\hline القيمة & البيــــــان & م \\
\hline من 80 الى 120 ملم & منوسط طول شعير ات الاكريليك & 1 \\
\hline 2.7 ديتيكس & دقة شعير ات الاكريليك & 2 \\
\hline $3.1: 2$ & المنانة جر ام / تكس & 3 \\
\hline 1.2 منرى & 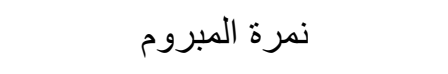 & 4 \\
\hline
\end{tabular}

ت 2 - 4 - مراحل الإنتاج:- Production Stages

تم تثغيل العينات على خط الغزل طبقاً للمراحل التالية:خط التفتيح والتنظيف ويتكون من Spinning Production Line

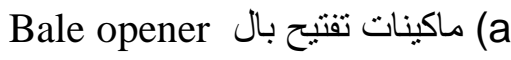

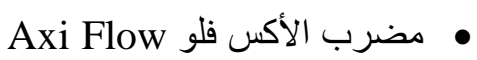
• الخلاط ذو الأربع غرف Multi Mixer • Beater RV مضرب • 3 Rollers Complete ماكينة ذات المضارب الثناثة • •

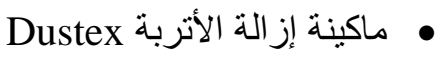
Carding M / c الكرد (b 
Drawing M / c/ I السحب ممر اول (c (d السحب ممر ثان Drawing M / c II جهاز السحب : 4/3 (e البرم Roving M /c نظام القطن f ماكينة البرم المستخدمه فى إنتاج مبروم البولى أكريليك بنظام الألياف الصناعية: RY الغزل Winding M / c التدوير (h

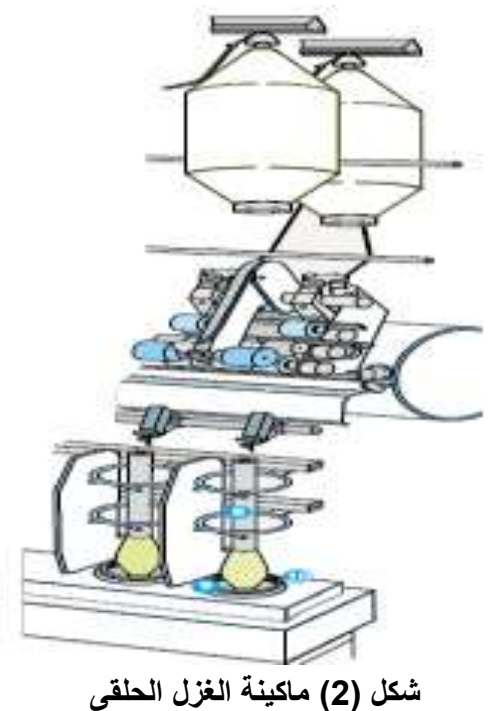

3 - النتائج و المناقشة: 3 - 1 - 1 - نتائج اختبارات والخنافيوط : جدول (3) يوضح نتائج الاختبار ات على الخيوط المنتجة عن طريق الخلط فى مرحلة الغزل الحلقى :

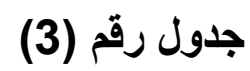
يوضح نتائج الاختبارات على الخيوط المنتجه عن طريق الخلط فى مرحله الغزل الحلقى

\begin{tabular}{|c|c|c|c|c|c|c|c|}
\hline التشعير & $\begin{array}{l}\text { Neps } \\
\% 140\end{array}$ & $\begin{array}{c}\text { الانتظامية } \\
\text { CV }\end{array}$ & القاطع & $\begin{array}{c}\text { الاستطالة } \\
\%\end{array}$ & $\begin{array}{l}\text { قوة الثد } \\
\text { " B.fcrce } \\
\text { "g.f }\end{array}$ & نسبة خطط المبروم & (تمرة الخيط \\
\hline 16.5 & 280 & 16.4 & 12.1 & 10.68 & 1117.3 & $1: 1$ & $1 / 7$ \\
\hline 16.1 & 185 & 13.7 & 14.1 & 9.8 & 1168.8 & $2: 1$ & $1 / 7$ \\
\hline 14.1 & 175 & 12.8 & 15.1 & 9.06 & 1208.5 & $3: 1$ & $1 / 7$ \\
\hline 15.1 & 550 & 17.4 & 10.2 & 9.52 & 614.4 & $1: 1$ & $1 / 10$ \\
\hline 12.6 & 325 & 14.2 & 13.4 & 9.37 & 690.8 & $2: 1$ & $1 / 10$ \\
\hline 12.5 & 240 & 13.7 & 14.9 & 8.05 & 745.1 & $3: 1$ & $1 / 10$ \\
\hline 12.3 & 695 & 19.8 & 9.5 & 8.79 & 432.3 & $1: 1$ & $1 / 14$ \\
\hline 11.3 & 535 & 15.5 & 13.1 & 7.47 & 509.3 & $2: 1$ & $1 / 14$ \\
\hline 11.1 & 520 & 14.2 & 13.8 & 7.3 & 611.4 & $3: 1$ & $1 / 14$ \\
\hline
\end{tabular}


3 - 1 - 1 - العلاقة بين نمرة الخيط ونسبة الخلط المختلفة للخيوط ومتوسط قوة الثد للخيوط المنتجة:

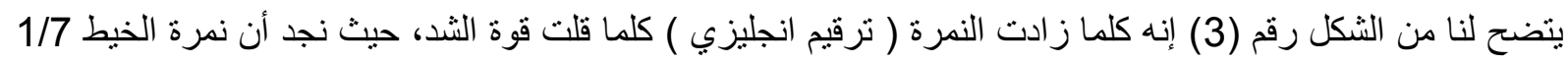

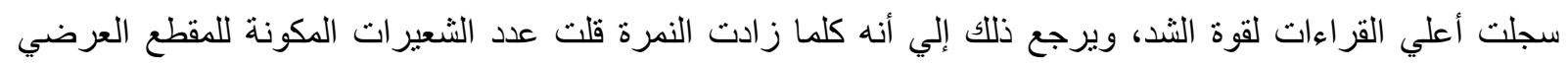

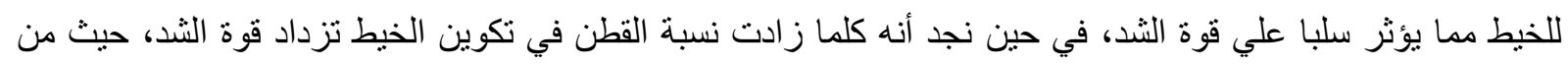

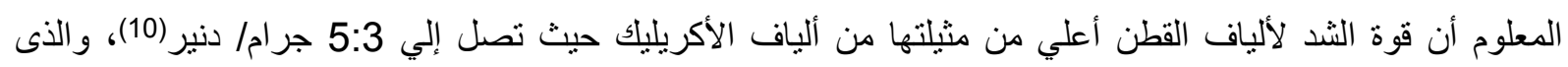
ينعكس إيجابياً على قوة شد الخيط الناتج.

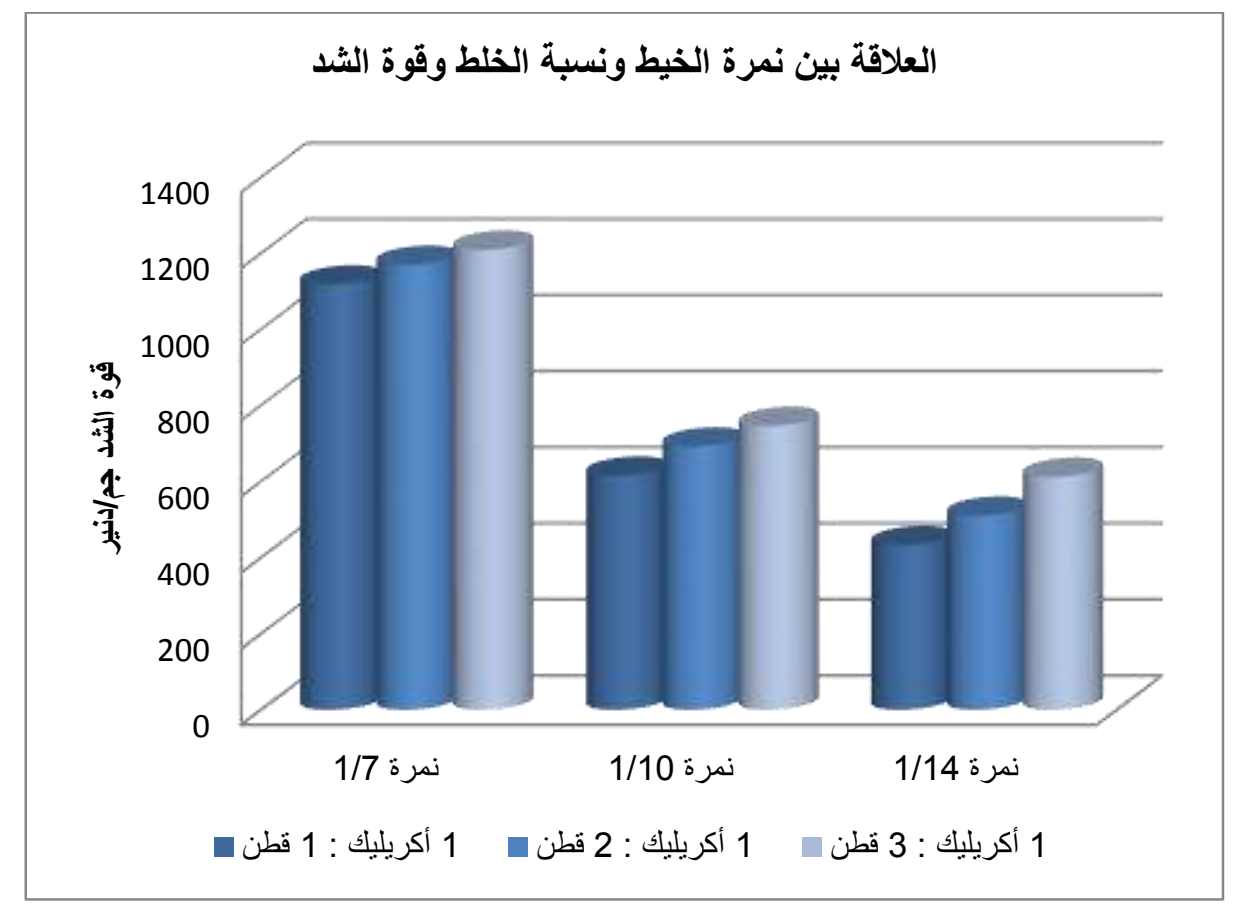

شكل (3) العلاقة بين نمرة الخيط ونسبة الخلط وقوة الثد

3 - 1 - 2 - العلاقة بين النمرة ونسبة الخلط المختلفة للخيوط و نسبة الاستطالة للخيوط المنتجة:

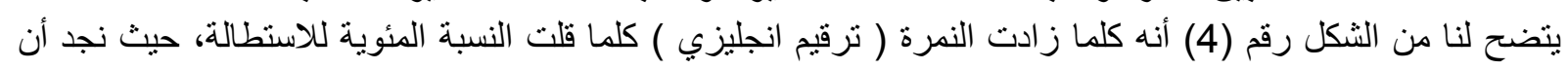
نمرة الخيط 1/7 سجلت أعلي القراءات ، ويرجع ذلك إلي أنه كلما زادت النمرة قلت عدد الثُعيرات المكونة للمقطع العرضي للخيط مما يؤثرسلباً علي النسبة المئوية للاستطالة،وذذلك لأن النمرة الرفيعة تحتوي على عدد كبير من البرمات

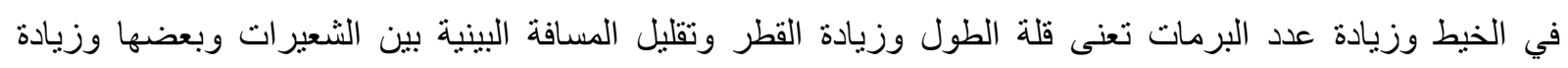

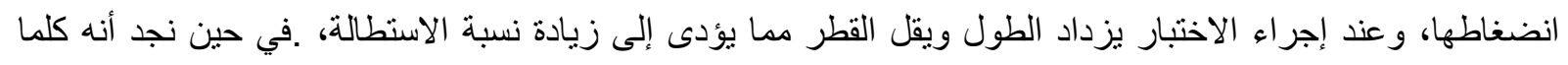

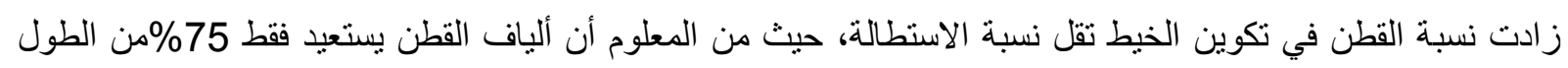
مع استطالة قدرها 2\%في حين تستطيع ألياف الاكريليك أن تستعيد 97\% من الطول الأصلى عند الاستطالة بنسبة 2\% 


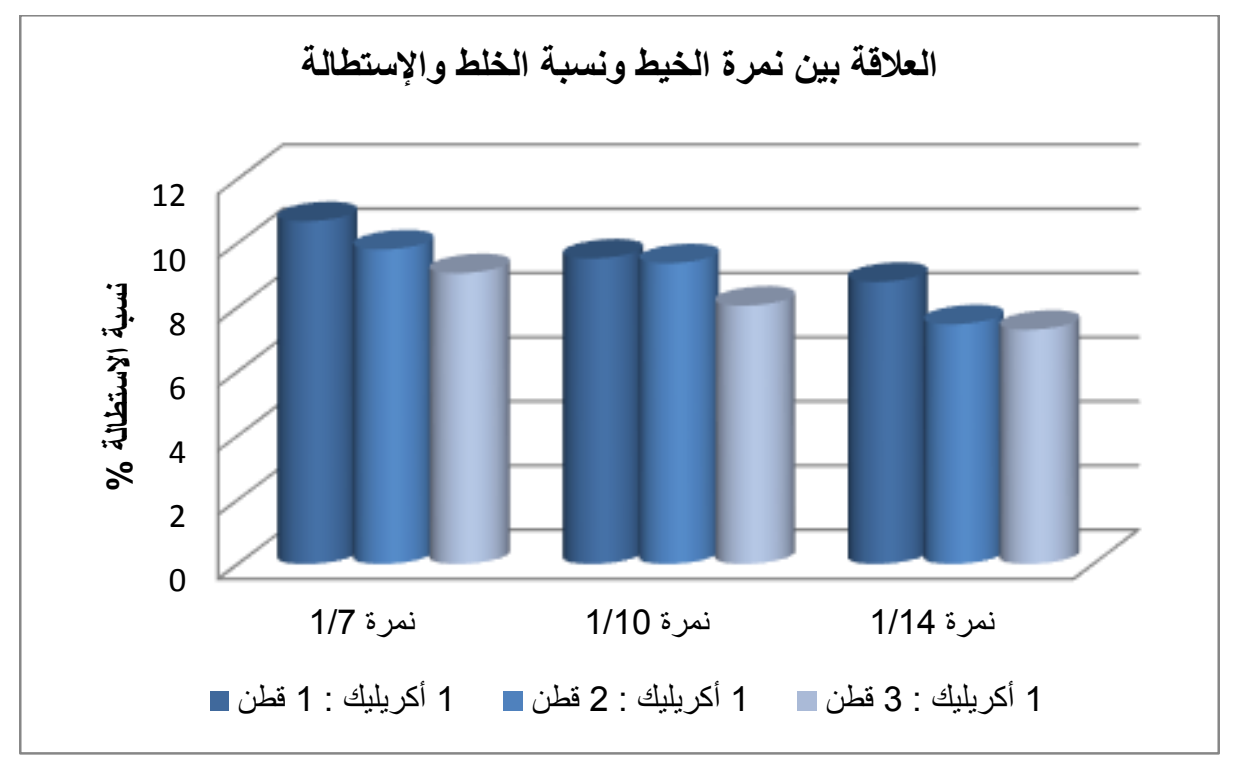

شكل رقم ( 4) الشكل يوضح العلاقة بين النمرة ونسبة الخلط المختلفة للخيوطو نسبة الإستطالة

3 - 1 - 3 - العلاقة بين النمرة ونسبة الخلط المختلفة للخيوط ومتوسط الحمل القاطع للخيوط المنتجة:

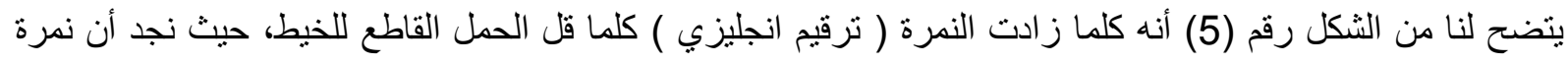
الخيط 1/7 سجلت أعلي القراءات للاختبار الحمل القاطع للخيط، ويرجع ذللك إلي أنه كلما زادت النمرة قلت عدد الثعير ات المكونة للمقطع العرضي للخيط مما يؤثرسلبا علي قراءات الحمل القاطع للخيط، في حين نجد إنه كلما زادت نسبة القطن في تكوين الخيط تزداد قر اءات الحمل القاطع للخيط، ومن المتعارف عليه إنه يوجد علاقة طردية ما بين قوة الثند و الحمل القاطع للخيط.

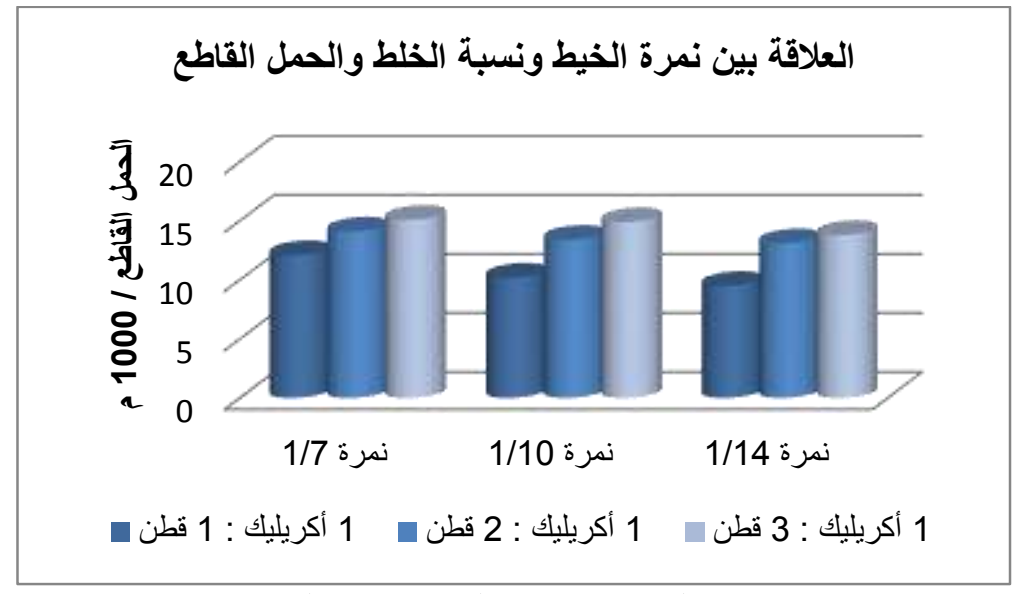

شكل رقم ( 5) الثكل يوضح العلاقة بين النمرة ونسبة الخلط المختلفة للخيوط ومتوسط الحمل القاطع

3 - 1 - 4 - العلاقة بين النمرة ونسبة الخلط المختلفة للخيوط وعدم الانتظامية للخيوط المنتجة:-

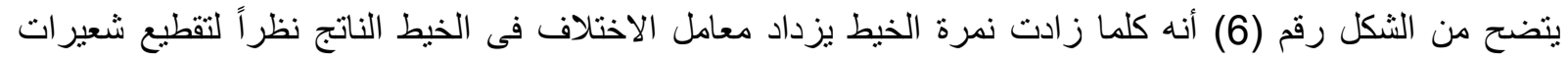
البولى اكريليك التى يبلغ أطوال شعيراتها 10 سم وتقطيعها بين زوج السلندرات بجهاز السحب بأطوال عشوائية غير منتظمة، فيختلف عدد شعيرات البولى اكريليك ويكون غير منتظم فيعطى عدم انتظامية أعلى والعكس صحيح، أما بالنسبة لزيادة مبروم القطن فيحدث خلطاً وزيادة التجانس أكثر فيعطى خيطاً أكثر انتظامية للخيط الناتج. 


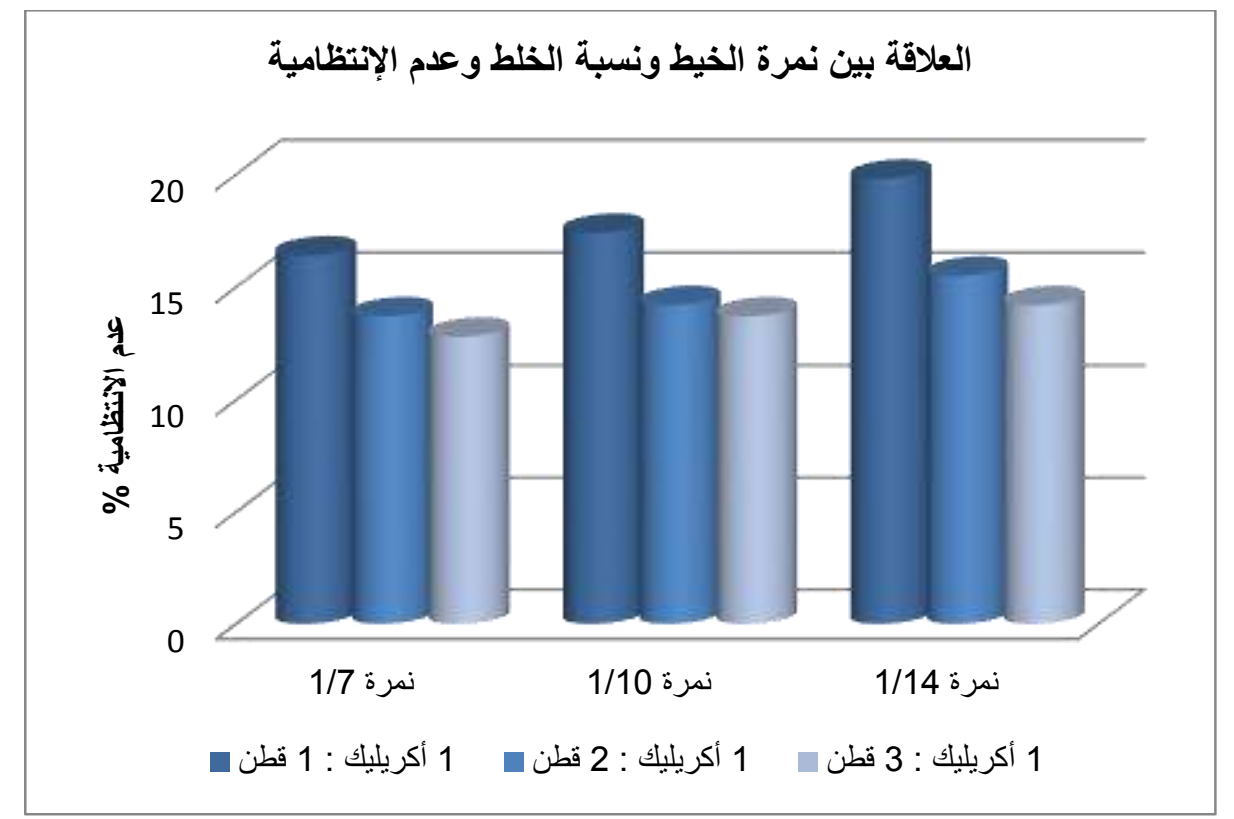

شكل رقم (6) الثكل يوضح العلاقة بين النمرة ونسبة الخلط المختلفة للخيوط وعدم الإتظامية

3 - 1 - 5 - العلاقة بين النمرة ونسبة الخلط المختلفة للخيوط ومتوسط عدد العقد للخيوط المنتجة:يتضح من الثكل رقم (7) أنه كلما زادت نمرة الخيط تزداد عدد العبد العقد في الخيط وبزيادة نسبة القطن فى الخيط المنتج يزداد نسبة التجانس وزيادة الخلط للخيط المنتج ويعطى عدداً أقل من العقد و العكس صحيح، وذلك بسبب تقطيع شعير ات البولى اكريليك بصفة عشوائية غير منتظمة فيتكون العقد وتكون أكثر للخيط السميك، و بزيادة مبروم القطن بالخيط (1 : 3 ) يزداد نسبة الخلط و التجانس بين شعير ات القطن المتقاربة في الطول فيعطى عدداً أقل للعقد فى الخيط الناتج و العكس.

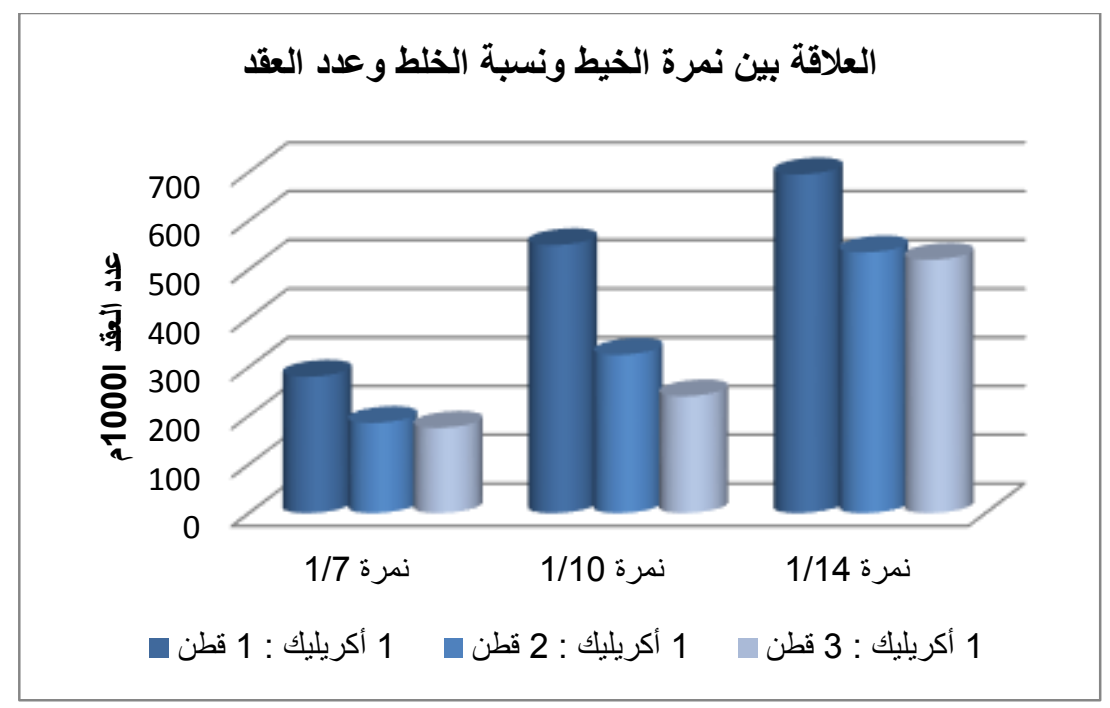

شكل رقم (7) الثكل يوضح العلاقة بين النمرة ونسبة الخلط المختلفة للخيوط ومنوسط عدد العقد

3 - 1 - 6 - العلاقه بين النمرة ونسبة الخلط المختلفة للخيوط ومتوسط التثعير للخيوط المنتجة:يتضح من الثكل رقم (8) أنه كلما زادت نمرة الخيط كلما زاد تشعير الخيوط و العكس كلما ز اد سمك الخيط كلما قل تشعير الخيط، وذلك لأن النمرة الأقل سككاً تستخدم قيم سحب أعلى بماكينة الغزل الحلقى مما يؤدى لزيادة اضطر اب الثعيرات،

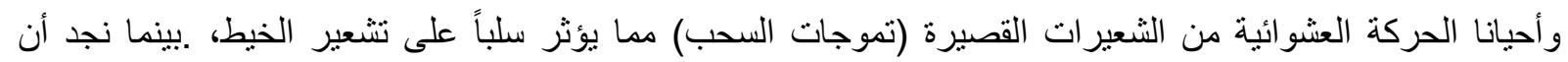


الخيط الذي ينكون من عدد مبروم بولى أكريليك :3 مبروم قطن يكون أكثر تجانساً فيعطى قراءات أقل والنالى يقل التشعير في الخيط الناتج .

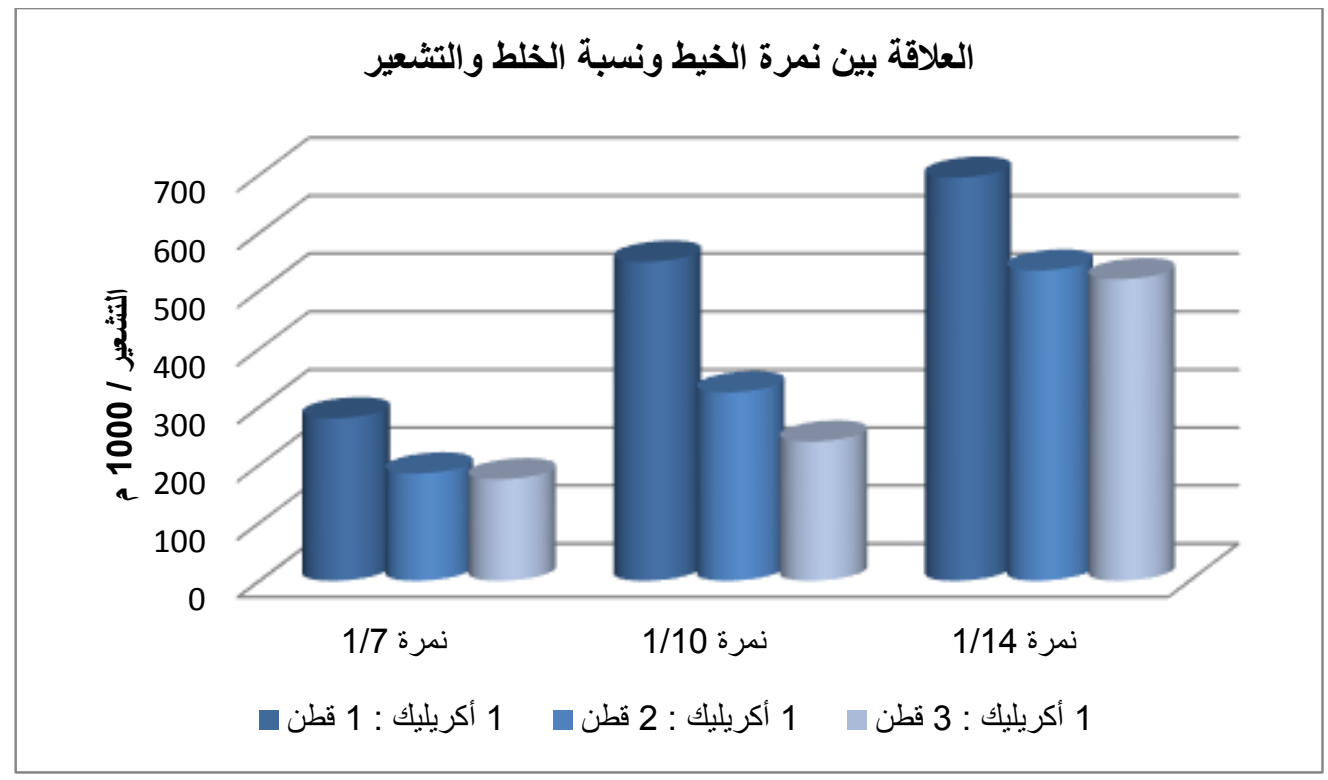

شكل رقم (8 ) الثكل يوضح العلاقة بين النمرة ونسبة الخلط المختلفة للخيوط ومتوسط التشعير

3 - 2 - 2 - ملخص النتائج:

1. كلما زادت نمرة الخيط كلما قلت قراءات قوة الثد و الحمل القاطع .، و عند زيادة نسبة الخلط بالقطن زادت قوة الثند و الحمل القاطع .

2. كلما زادت النمرة ( ترقيم انجليزي ) كلما قلت النسبة المئوية للاستطالة، و عند زيادة نسبة القطن في تكوين الخيط تقل نسبة الاسنطالة . 3. كلما كانت نمرة الخيط رفيعة كلما زادت عدم الانتظامية وعدد العقد و التشعير . .

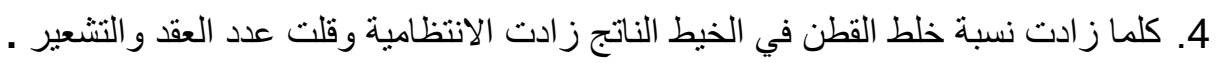

1. عبد السلام , محمد السيد: " تكنولوجيا الغزل-الجزء الاول" ، الهيئة العامة للكتاب, 1984. 1 - Eabd alsalam ,muhamad alsyd: " tkanulwjy aalghazla- aljuz' al'awla" , al hayyata leamat lilkitab , 1984.

$$
\text { 2.سالمان ,احمد علي: " ألياف النسيج", الهيئة العامة للكتاب, 1985م. }
$$

2 - Salman , 'ahmad ealay: "a liaf alnasij" , al hayyat aleamat lilkitab , 1985 m.

$$
\text { 3.سلطان , محمد أحمد : " ألياف النسيج ", منشأة المعارف , 1990م . }
$$

3 -Sultan ,muhamad 'ahmd: "aliaf alnasij" , munsha' atalmaearif , 1990 m.

4. عمر ,جمال مصطفى كمال علوان : " دراسة تاثثر هجرة الثعيرات على الخواص الطبيعية والميكانيكية لبعض الخيوط

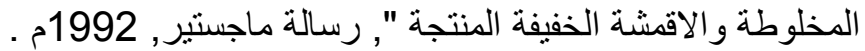

4 -Oumar ,jamal mustafaa kamal ealwan: "drrastta thir hijrat alshaeirat ealaaalk huwwasaltabieiatwalmikanikiatlibaedalkhuyutalmakhlutatwalaqmshtalkhafifa" risalatmajstyr , $1992 \mathrm{~m}$. 
المجلد الخامس - العدد الثاني والعشرون

5.صابر , صلاح الدين : "صناعة الغزل والمنسوجات", مركز تطوير الصناعات النسيجية , البرامج التدريبية النسيجية, 2004

5 -Sabir ,salahaldyn: "isnaeatalghazlwalmansujat" , markaztatwiralsinaeatalnasijiat , albaramijaltadribiatalnasijiat, $2004 \mathrm{~m}$.

6.السيد ,فتحي اسماعيل ,إبراهيم مجدي عبد الرحمن:"الالياف والخيوط الصناعية" , مركز المعلومات, صندوق دعم صناعة الغزل و المنسوجات, الإسكندرية ,2005م.

6 - Alsyd ,fathi 'iismaeil , 'iibrahim majdie abdalrahmn: "alalyaf walkhuyutalsinaeia" , markazalmaelumat, sunduqdaemsinaeatalghazlwalmansujat, al'iiskandariat, $2005 \mathrm{~m}$.

7. عمارة , وفاء محمد مصطفى: "دراسة بعض العو امل المؤثرة على هجرة الثعيرات وتاثثير ها على الخواص الفيزيقية

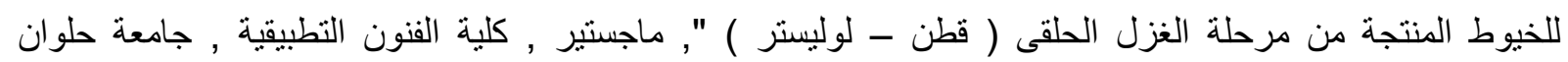
2010,

7 - Eamarat, wafa' muhamad mustafa: "drast bed aleawamil almuatharat ealaa hijrat alshaeirat watahdidiha ealaa alkhawas

8.الطنطاوى ، سمير أحمد ، سيدعلى السيد :" تكنولوجيا الغزل الحديثة ", الثنهابى للطباعة والنشر ، 2011 م .

8 - Tantawi , Samir ahmad, sydealaaalsyd "tknwlwjya 'intaj alkhywt", alshinhabi liltibaeat walnishri,, ( 2011 ) .

9.ياسر محمد عيد حسن : : كلية التصاميم ـ قسم تصميم الازياء - جامعة ام القرى ـ المملكة العربية السعودية - 2011 -

9- Hassan Yaser Mohamed Eed : kolet al takem - kasem al azyaa - gamet om al kora - al mmlka al arba el sodea - $2011 "$

10.إسماعيل, محمد صبري:"خامات النسيج", كلية الفنون التطبيقية ,2015م.

10- 'Iismaeil, muhamadsabry: "khamatalnasij", kuliyata lfunun altatbiqiat, 2015 m.

11.الطنطاوي , سمير أحمد: "تكنولوجيا إنتاج الخيوط",الثنهابي للطباعة و النشر, 2016 "2016.

11- Altantawiu, samir ahmd: "tknwlwjya 'intaj alkhywt", alshinhabi liltibaeat walnishri, 2016 m.

12.معبد , حسين سيد علي :" إمكانية تشغيل القطن البوركيني بمصانع الغزل المصرية وتأثير ذلك على خواص الخيوط

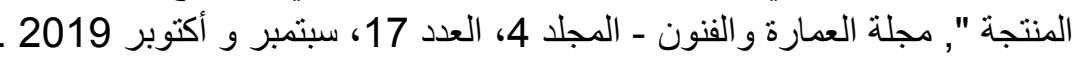

12 - maebad, husayn syd eali:" 'iimkaniat tashghil alqatn alburkinii bimasanie alghazl almisriat watathir dhlk ealaa khawas alkhuyut almuntija ", majalat aleamarat walfunun almujalid 4, aleadad 17, sibtambar w 'uktubar 2019.

13.الجمل, فيروز أبو الفتوح :" نأثير بعض التراكيب البنائية لجاكارد نريكو اللحمة على بعض الخواص الوظيفية لأقشة

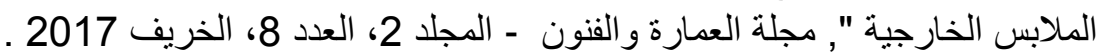

13 - aljml, firuz 'abu alfutuh :" tathir bed altarakib albinayiyat ljakard trykw alluhmat ealaa bed alkhawwas alwazifiat li'aqmshat almalabis alkharijia ", majalat aleamarat walfnunalmujalid 2, aleadad 8, alkharif 2017 .

ثانيا : المراجع الأجنبية :

14.Bogdan, J.F., "Measurement of Fiber Mixing in Yarn" extile Research Journal, No.5, "1970", PP. (415).

15.Bernard, P.Corbman: Textiles Fiber,Mc Crow Hill book Company,NewYork,1983.

16.Construction", The Textile Institute, Manchester, England,1973.

17.J.W.S. Hearle, B.S. Gupta and V.B . Merchant, , Text, Res . J . Vol.35 , 1965 , April , pp . $329-334$.

18.p,p Townend, Ciba Review , No . 3, 1965 , pp . 16-21

19.Composed of Yarns Spun on the Cotton System "Textile Research Journal, No. 2, "1984",PP.(82). 